\title{
Solving Signal Control Problems with Second-Order Sensitivity Information of Equilibrium Network Flows
}

\author{
Hsun-Jung Cho and You-Heng Huang \\ Department of Transportation and Logistics Management, National Chiao Tung University, Hsinchu 30010, Taiwan \\ Correspondence should be addressed to Hsun-Jung Cho; hjcho001@gmail.com
}

Received 24 November 2013; Revised 3 April 2014; Accepted 5 April 2014; Published 11 May 2014

Academic Editor: Kazutake Komori

Copyright ( 2014 H.-J. Cho and Y.-H. Huang. This is an open access article distributed under the Creative Commons Attribution License, which permits unrestricted use, distribution, and reproduction in any medium, provided the original work is properly cited.

The equilibrium network signal control problem is represented as a Stackelberg game. Due to the characteristics of a Stackelberg game, solving the upper-level problem and lower-level problem iteratively cannot be expected to converge to the solution. The reaction function of the lower-level problem is the key information to solve a Stackelberg game. Usually, the reaction function is approximated by the network sensitivity information. This paper firstly presents the general form of the second-order sensitivity formula for equilibrium network flows. The second-order sensitivity information can be applied to the second-order reaction function to solve the network signal control problem efficiently. Finally, this paper also demonstrates two numerical examples that show the computation of second-order sensitivity and the speed of convergence of the nonlinear approximation algorithm.

\section{Introduction}

The network signal control problem (NSCP) is to find the optimal signal setting which improves the performance of existing facilities in a transportation network. Conventional methods for optimizing signal settings can be divided into two types: stage-based and group-based approaches [16]. The stage-based approach divided the signal cycle into separate stages and solved the optimal signal settings for each group of compatible traffic movements in stages. This approach is regarded as superior in the concern for safety and loss of capacity with phase switching [6]. The group-based approach considered each group of traffic streams having right-of-way in the time domain directly. Compared with the stage-based approach, the group-based approach has a higher degree of flexibility in signal timing arrangement $[3,7]$. However, the most optimization models proposed so far usually converged to a local optimal solution and without taking traffic rerouting effects into account when solving NSCP [5]. The equilibrium network signal control problem (ENSCP) is used to find an optimal network signal design when the network flow pattern is constrained to be equilibrium. Friesz [8] points out that this is a problem of interest because of Braess' paradox [9]. This paradox shows that the congestion of the network may be severer when adding capacity to a congested network without taking the reaction of network users into consideration. Hence, in practice, the equilibrium network signal design problem must be solved by constraining the network flow pattern to meet user equilibrium. The user equilibrium network design with fixed transportation demand has been studied in both discrete [10] and continuous [11] versions. To help solve the signal control problem, Allsop [12] pointed out that the route choices of road users should be considered the impacts of signal settings changing. Gartner et al. [13] and Fisk [14] described the signal control problem as a Stackelberg or leader-follower game between road users and the administration. The Stackelberg game can be represented as a bilevel problem, where the upper-level problem aims to find the optimal signal setting or link capacity expansions which maximizes system performance, and the lower-level problem aims to solve the user equilibrium (UE) flows, respectively $[15,16]$.

Marcotte [17], Sheffi and Powell [18], Heydecker and Khoo [19], Smith and van Vuren [20], Tan et al. [21], Cantarella et al. [22], Gartner et al. [13], Smith et al. [23], van Vuren and van Vliet [24], and others proposed algorithms to solve the network problem. However, when calculating optimal settings in general road networks, there were no efficient solution algorithms that are combined with anticipating the reactions of road users. Moreover, the iterative optimization 
assignment (IOA) procedure which solves signal settings and equilibrium flows cannot be expected to converge to the true solution and might lead to a decline in network performance $[14,25]$. The sensitivity analysis-based algorithm evaluates the influence factors as the derivatives of the reaction functions with respect to the upper-level decision variables. The derivative information is obtained by implementing sensitivity analysis for a given solution of the user equilibrium problem [26-30]. For the singularity, algorithms can only solve a small network problem. Cho [31] proposed a generalized inverse method, Cho et al. [32] proposed a row reduction method, Patriksson [33], Josefsson, and Patriksson [34] proposed directional derivative method, and Yang and Bell [35] proposed a column reduction method to overcome the singularity problem. In the sensitivity analysis-based linear approximation algorithm, the sensitivity information is used to create a linear approximation of the reaction function and is then inserted into the upper-level problem, iterated until the solutions converge $[34,36,37]$. Recently, Chiou has conducted several studies related to optimal design of area traffic control with equilibrium network flows and proposed a number of computational algorithms to solve the problem, such as the projected Quasi-Newton method and the bundle subgradient projection method [38-41]. Moreover, the ENSCP, combined with an explicit traffic model, TRANSYT, was proposed to evaluate the performance index of the system more precisely [4, 38, 42-44].

The remainder of this paper is organized as follows: Section 2 introduces the equilibrium network flow models and the first-order sensitivity formula obtained by row reduction method. Section 3 introduces the matrix calculus theory and the second-order sensitivity formula. The network signal control model and solution algorithm are presented in Section 4. Finally, a numerical example and conclusion are presented in Sections 5 and 6, respectively.

\section{Sensitivity Analysis of Equilibrium Network Flows}

2.1. Equilibrium Network Flow Models. Consider a transportation network consisting of a finite set of nodes $i \in N$ and a finite set of $\operatorname{arcs} a \in A$ together with a nonempty set of origin-destination (OD) pairs $w \in W$. For each $w \in W$, there is a nonempty finite set of paths, $p \in P_{w}$. Let real numbers, nonnegative reals, and positive reals be denoted by $R, R_{+}$, and $R_{++}$, respectively. The path flow vector, $h$, arc flow vector, $f$, and travel demand vector, $T$, are denoted in the following equations:

$$
\begin{gathered}
h=\left(h_{p}: p \in P, P=\bigcup_{w \in W} P_{w}\right) \in R_{+}^{|P|}, \\
f=\left(f_{a}: a \in A\right) \in R_{+}^{|A|}, \\
T=\left(T_{w}: w \in W\right) \in R_{++}^{|W|},
\end{gathered}
$$

where $|P|,|A|$, and $|W|$ denote the cardinalities of $P, A$, and $W$, respectively. The relationship between arc flow, path flow, and travel demand is given by

$$
\Delta h=f, \quad \Lambda h=T,
$$

where $\Delta$ is a $|A| \times|P|$ matrix, with $\Delta_{a p}=1$, if $\operatorname{arc} a$ belongs to path $p$ and $\Delta_{a p}=0$ otherwise; $\Lambda$ is a $|W| \times|P|$ matrix, with $\Lambda_{w p}=1$, if OD pair $w$ belongs to path $p$ and $\Lambda_{w p}=0$ otherwise. In general, $\Delta$ is called arc/path incidence matrix and $\Lambda$ is called $\mathrm{OD} /$ path incidence matrix.

In sensitivity analysis, a vector of perturbation parameters with dimension $\rho, s \in R^{\rho}$, is introduced. The arc cost function and travel demand function are supposed to be influenced by $s$. Let $t(f, s)$ be the arc cost function vector and let $T(s)$ be the travel demand function vector. The path cost function vector is given by $c(h, s)=\Delta^{T} t(f, s)$. When the network equilibrium is reached, the following equations must be satisfied:

$$
\begin{aligned}
& h_{p}>0 \Longrightarrow c_{p}(h, s)=\mu_{w}, \quad p \in P_{w}, w \in W, \\
& h_{p}=0 \Longrightarrow c_{p}(h, s) \geq \mu_{w}, \quad p \in P_{w}, w \in W,
\end{aligned}
$$

where $\mu_{w}$ is the equilibrium path cost of OD pair $w$. Equations (3) are recognized as Wardrop equilibrium conditions; say, there is no traveler can change path unilaterally to improve his travel time [45]. Generally, the equilibrium network flow problem can be written in the form of variational inequality (VI) problem as follows [46]. Find $f \in \Omega(s)$ such that

$$
\begin{gathered}
t(f(s), s)^{T}(u-f) \geq 0, \quad \forall u \in \Omega(s), \\
\Omega(s)=\{f \mid \Delta h=f, \Lambda h=T(s), h \geq 0\},
\end{gathered}
$$

where $\Omega$ is the feasible arc flow solution set of the network flow problems.

An equivalent VI can be written with the cost function in terms of path flow variable $h$ rather than arc flow variable $f$ as follows. Find $h \in \Gamma(s)$ such that

$$
\begin{gathered}
c(h, s)^{T}(u-h) \geq 0, \quad \forall u \in \Gamma(s), \\
\Gamma(s)=\{h \mid \Lambda h=T(s), h \geq 0\},
\end{gathered}
$$

where $\Gamma$ is the feasible path flow solution set of the network flow problems.

\subsection{First-Order Sensitivity Formula for Equilibrium Network} Flows. The classical first-order sensitivity analysis for equilibrium network flows was proposed by Tobin and Friesz [47]. However, Tobin and Friesz method had a strong requirement on the topology of network which may not hold in practical networks [48]. Cho et al. proposed the row reduction method to overcome this issue [32]. In this paper, we only summarize the key results of the row reduction method, and the readers are encouraged to refer to the original paper [32] for more details.

In the row reduction method, a maximal set of rows from $\Delta$, say $\Delta_{1}$, is selected for which the combined matrix $\left[\Delta_{1}, \Lambda\right]^{T}$ is of full row rank. Hence, $\Delta$ can be partitioned as $\left[\Delta_{1} ; \Delta_{2}\right]$. Assume that the number of independent arcs is $\alpha_{1}$ and the number of dependent arcs is $\alpha_{2}$, respectively. Therefore, $|A|=$ $\alpha=\alpha_{1}+\alpha_{2}$. For a differentiable function, $f: S \rightarrow R^{m}$, let the partial derivative of $f$ with respect to $s$ (the Jacobian matrix of $f$ ) be denoted by $D_{s} f$. Let the second-order partial 
derivative of $f$ with respect to $s$ be denoted by $H_{s} f$. The firstorder sensitivity formula can be expressed as

$$
\left[\begin{array}{c}
D_{s} f(s) \\
D_{s} \mu(s)
\end{array}\right]=\left[\begin{array}{cc}
D_{f} t(f, s) & -M^{T} \\
M & 0
\end{array}\right]^{-1}\left[\begin{array}{c}
-D_{s} t(f, s) \\
\Delta_{2} N_{2} D_{s} T(s)
\end{array}\right],
$$

where

$$
\begin{gathered}
M=\left[\begin{array}{ll}
\Delta_{2} N_{1} & -I
\end{array}\right], \\
N_{1}=\Delta_{1}^{T} M_{11}+\Lambda^{T} M_{21}, \\
N_{2}=\Delta_{1}^{T} M_{12}+\Lambda^{T} M_{22}, \\
\left(\left[\begin{array}{c}
\Delta_{1} \\
\Lambda
\end{array}\right]\left[\begin{array}{c}
\Delta_{1} \\
\Lambda
\end{array}\right]^{T}\right)^{-1}=\left[\begin{array}{ll}
M_{11} & M_{12} \\
M_{21} & M_{22}
\end{array}\right] .
\end{gathered}
$$

\section{Second-Order Sensitivity Formula for Equilibrium Network Flows}

The sensitivity analysis-based nonlinear approximation heuristic algorithm (NLAA) was firstly proposed by Cho and Lin [37]. With the first-order and the second-order sensitivity information, the reaction function of the lower-level problem can be approximated by a nonlinear function. However, Cho and Lin did not provide the general form of the second-order sensitivity information. In this section, we will introduce the preliminary of matrix calculus theory and derive the general form of the second-order sensitivity formula for equilibrium network flows.

3.1. Preliminary Definitions and Theorems. To derive the second-order sensitivity formula, we introduce some definitions and theorems of matrix calculus as follows $[49,50]$.

Definition 1 (Kronecker product). Let $U$ be an $m \times n$ matrix and let $V$ be a $p \times q$ matrix; then the Kronecker product of $U$ and $V$, denoted by $U \otimes V$, is an $m p \times n q$ matrix defined by

$$
U \otimes V=\left[\begin{array}{ccc}
u_{11} V & \cdots & u_{1 n} V \\
\vdots & \ddots & \vdots \\
u_{m 1} V & \cdots & u_{m n} V
\end{array}\right]
$$

Definition 2 (Vector operator). Let $U$ be an $m \times n$ matrix and $U_{j}$ is the $j$ th column of $U$; then vec $U$ is the $m n \times 1$ vector:

$$
\operatorname{vec} U=\left[\begin{array}{c}
U_{1} \\
U_{2} \\
\vdots \\
U_{n}
\end{array}\right]
$$

Definition 3. Let $U$ be an $m \times n$ real matrix function of a $p \times q$ matrix of real variables $s$. The derivative of $U$ with respect to $s$ is the $m n \times p q$ matrix:

$$
D_{s} U=\frac{\partial \operatorname{vec} U}{\partial(\operatorname{vec} s)^{T}}
$$

Theorem 4 (chain rule for matrix functions, Magnus and Neudecker, 1985 [49]). Let $S$ be a subset of $R^{m \times n}$ and assume that $U: S \rightarrow R^{p \times q}$ is differentiable at an interior point $y$ of $S$. Let $P$ be a subset of $R^{p \times q}$ such that $U(x) \in P$ for all $x \in S$ and assume that $V: P \rightarrow R^{r \times s}$ is differentiable at an interior point $z=U(y)$ of $P$. Then the composite function $F: S \rightarrow R^{r \times s}$ defined by $F(x)=V(U(x))$ is differentiable at $y$ and

$$
D_{y} F=\left(D_{z} V\right)\left(D_{y} U\right) \text {. }
$$

Theorem 5 (Magnus and Neudecker, 1985 [49]). Let $U: S \rightarrow$ $R^{m \times r}$ and $V: S \rightarrow R^{r \times n}$ be two matrix functions defined and differentiable on an open set $S$ in $R^{p \times q}$. Then the simple product $U V$ is differentiable on $S$ and the Jacobian matrix is the $m n \times p q$ matrix:

$$
D_{s}(U V)=\frac{\partial \operatorname{vec} U V}{\partial(\operatorname{vec} s)^{T}}=\left(V^{T} \otimes I_{m}\right) D_{s} U+\left(I_{n} \otimes U\right) D_{s} V
$$

where $I_{m}$ and $I_{n}$ are the identity matrices of size $m$ and $n$, respectively.

Theorem 6 (Magnus and Neudecker, 1999 [50]). Let $f: S \rightarrow$ $R^{m}$ be a function defined on a set $S$ in $R^{n}$. Let $r$ be an interior point of $S$ and let $B\left(s_{0} ; r\right)$ be an $n$-ball lying in $S$. Let $s$ be a point in $R^{n}$ with $\|s\|<r$, so that $s_{0}+s \in B\left(s_{0} ; r\right)$. If $f$ is twice differentiable at $s_{0}$, then the second-order Taylor expansion of function $f$ at $s_{0}+s$ is

$$
f\left(s_{0}+s\right)=f\left(s_{0}\right)+d f\left(s_{0} ; s\right)+\frac{1}{2} d^{2} f\left(s_{0} ; s\right),
$$

where $d f\left(s_{0} ; s\right)$ and $d^{2} f\left(s_{0} ; s\right)$ are the first differential and the second differential of $f$ at $s_{0}$, respectively, and

$$
\begin{gathered}
d f\left(s_{0} ; s\right)=D_{s} f\left(s_{0}\right) \cdot\left(s-s_{0}\right) \\
d^{2} f\left(s_{0} ; s\right)=\left(\left(s-s_{0}\right)^{T} \otimes I_{m}\right) \cdot H_{s} f\left(s_{0}\right) \cdot\left(s-s_{0}\right) .
\end{gathered}
$$

3.2. Second-Order Sensitivity Formula for Equilibrium Network Flows. To derive the second-order sensitivity formula for equilibrium network flows, it is intuitive to take derivative of (6) with respect to $s$. For convenience, let

$$
\begin{gathered}
{\left[\begin{array}{cc}
D_{f} t(f(s), s) & -M^{T} \\
M & 0
\end{array}\right]^{-1}=U(f(s), s),} \\
{\left[\begin{array}{c}
-D_{s} t(f(s), s) \\
\Delta_{2} N_{2} D_{s} T(s)
\end{array}\right]=V(f(s), s),}
\end{gathered}
$$

where $U$ is an $\left(\alpha+\alpha_{2}\right) \times\left(\alpha+\alpha_{2}\right)$ matrix and $V$ is an $\left(\alpha+\alpha_{2}\right) \times \rho$ matrix, respectively.

Lemma 7. The second-order sensitivity for equilibrium network flows is

$$
\left[\begin{array}{l}
H_{s} f \\
H_{s} \mu
\end{array}\right]=\left(V^{T} \otimes I_{\left(\alpha+\alpha_{2}\right)}\right) D_{s} U+\left(I_{k} \otimes U\right) D_{s} V,
$$


where

$$
\begin{gathered}
U(f(s), s)=\left[\begin{array}{cc}
D_{f} t(f(s), s) & -M^{T} \\
M & 0
\end{array}\right]^{-1}, \\
V(f(s), s)=\left[\begin{array}{c}
-D_{s} t(f(s), s) \\
\Delta_{2} N_{2} D_{s} T(s)
\end{array}\right] .
\end{gathered}
$$

Proof. Since the first-order sensitivity is the product of (17), the second-order sensitivity can be obtained by taking derivative of the product with respect to $s$ directly. According to Theorem 5 , the formula of the second-order sensitivity is expressed as (16) and the proof is complete.

\section{Network Signal Control Model and Solution Algorithm}

4.1. Network Signal Control Model. Consider the signal optimization problem, where the aim of the regulating agency is to minimize a network performance function $Z(s)$ such as total travel time or gas consumption, with fixed OD travel demand, where travelers select routes on the network in an optimal user fashion. Notably, $S$ denotes the set of feasible signal control variables. For any given $s \in S$, a user optimal arc flow solution $f(s) \in \Omega$ exists and the problem of the regulator is to solve

$$
\begin{aligned}
P_{1}: & \min _{s \in X_{1}} \\
& Z(s)=P(f(s), s) \\
\text { s.t. } & \text { user equilibrium. }
\end{aligned}
$$

In the general problem, the signal variable that can be set by the controlling agent is green time. By specifying the cost functions $t_{a}$ for each network arc $a$ in terms of these variables and assuming that the behavioral hypothesis for route choice follows the first principle of Wardrop [45], problem $P_{1}$ can be presented as

$$
\begin{aligned}
P_{2}: & \min _{s \in X_{1}} Z(s)=\sum_{a \in A} t_{a}(f(s), s) f_{a}(s) \\
\text { s.t. } & t(f(s), s) \cdot(u-f) \geq 0, \quad \forall u \in \Omega .
\end{aligned}
$$

If $t(s, f)$ is strictly monotone, then, for each $s \geq 0,(20)$ has a unique solution and function $f(s)$ is (continuously) differentiable at every point $s \geq 0$. Thus, $P_{2}$ can be rewritten as $P_{3}$ :

$$
\begin{gathered}
P_{3}: \min _{s \in X_{1}} Z(s)=\sum_{a} t_{a}(f(s), s) f_{a}(s) \\
\text { s.t. } \quad s \geq 0 .
\end{gathered}
$$

Also, given $R(s, f(s))=\min t(f(s), s) \cdot(u-f)$, then $P_{2}$ is equivalent to $P_{4}$ :

$$
\begin{array}{cl}
P_{4}: & \min _{s \in X_{1}} \\
\text { s.t. } & R(s)=\sum_{a} t_{a}(f(s), s) f_{a}(s) \\
&
\end{array}
$$

4.2. Solution Algorithms. The iterative optimization assignment (IOA) method described by Tan et al. [21] is proceeded as follows. First, fix $s$ and solve (20) for $f$; then fix $f$ and solve (19) for $s$, continuing this process until $s^{k+1}-s^{k} \rightarrow 0$ or $f^{k+1}-f^{k} \rightarrow 0$. The final solution $\left(f^{N}, s^{N}\right)$ is termed the Nash solution. Notably, that the solution obtained using the IOA algorithm is not necessarily an optimal solution of the equilibrium network control problem [14]. The sensitivity analysis of equilibrium network flows [32, 47] was used to solve the equilibrium network signal design problem $[26,36,51]$.

4.2.1. A Sensitivity Analysis-Based Linear Approximation Heuristic Algorithm. The challenge in solving problem $P_{2}$ is that, since the lower level of the problem cannot be represented in closed form, it is impossible to obtain an explicit reaction function that can be plugged into the upper level. In the sensitivity analysis-based linear approximation heuristic algorithm, the sensitivity information is used to create a linear approximation of the reaction function and is then inserted into the upper-level problem, iterated until the solutions converge (abbreviated as LAA) [36].

The heuristic is detailed as follows.

\section{Algorithm A1.}

Step 0. Determine a fixed small value $\delta>0$ and an initial value $s^{0}$. Set $k=0$.

Step 1. Solve (18) given $s^{k}$ and yielding $f^{k}$.

Step 2. Calculate the sensitivity information $D_{s} f$ by (6).

Step 3. Using $D_{s} f$, Taylor expansion and Theorem 6 form the linear approximation $f^{k+1}, f^{k+1}=f^{k}+D_{s} f \cdot\left(s^{k+1}-s^{k}\right)$. Since $f^{k}, s^{k}$, and $D_{s} f$ are known, $f^{k+1}$ can be replaced by a function of $s^{k+1}$. Thus, $f^{k+1}=A+B s^{k+1}$.

Step 4. Reformulate (21) as

$$
\begin{aligned}
& \min _{s} \sum_{a} t_{a}\left(A+B s^{k+1}, s\right) \cdot\left(A+B s^{k+1}\right) \\
& \text { s.t. } \quad s \geq 0 .
\end{aligned}
$$

Step 5. Solve the problem in Step 4 using any software package which can solve the optimal solution for $s^{k+1}$. If $\left|s^{k+1}-s^{k}\right| \leq \delta$, then stop; otherwise set $k=k+1$ and go to Step 1 .

4.2.2. A Sensitivity Analysis-Based Nonlinear Approximation Heuristic Algorithm. In the sensitivity analysis-based linear approximation heuristic algorithm, the reaction function of the lower level is based on approximation by a linear function. In this section, the reaction function of the lower-level problem is based on approximation by a nonlinear function and is plugged into the upper-level problem and is iterated until the solutions converge (abbreviated as NLAA) [37].

\section{Algorithm A2.}

Step 0. Determine a fixed small value $\delta>0$ and an initial value $s^{0}$. Set $k=0$. 
Step 1. Solve (20) given $s^{k}$ and yielding $f^{k}$.

Step 2. Calculate the sensitivity information $D_{s} f$ and $H_{s} f$ by (6) and (16).

Step 3. Using $D_{s} f$ and $H_{s} f$, Taylor expansion and Theorem 6 form the nonlinear approximation $f^{k+1}$ :

$$
\begin{aligned}
f^{k+1}= & f^{k}+D_{s} f \cdot\left(s^{k+1}-s^{k}\right)+\frac{1}{2} \cdot\left(\left(s^{k+1}-s^{k}\right)^{T} \otimes I_{m}\right) \\
& \cdot H_{s} f \cdot\left(s^{k+1}-s^{k}\right) .
\end{aligned}
$$

Since $f^{k}, s^{k}, D_{s} f$, and $H_{s} f$ are known, $f^{k+1}$ can be replaced by a function of $s^{k+1}$. Thus, $f^{k+1}=A+B s^{k+1}+C\left(s^{k+1}\right)^{2}$.

Step 4. Reformulate (21) as

$$
\begin{gathered}
\min _{s} \sum_{a} t_{a}\left(A+B s^{k+1}+C\left(s^{k+1}\right)^{2}, s\right) \\
\cdot\left(A+B s^{k+1}+C\left(s^{k+1}\right)^{2}\right)
\end{gathered}
$$

s.t. $\quad s \geq 0$.

Step 5. Solve the problem in Step 4 using any software package which can solve the optimal solution for $s^{k+1}$. If $\left|s^{k+1}-s^{k}\right| \leq \delta$; then stop; otherwise set $k=k+1$ and go to Step 1 .

In addition to describing the algorithm in more detail, we will provide a proof that if this algorithm converges, it converges to an optimal solution of problem $P_{2}$.

Lemma 8. If algorithm A2 converges, it converges to a critical point of $P_{2}$.

Proof. If the sequence $s^{k}$ converges to $s^{*}, s^{k} \rightarrow s^{*}$, then we know that

(1) if we set $s^{0}=s^{*}, f^{0}=f^{*}$, then $s^{1}=s^{*}, f^{1}=f^{*}$; and

(2) let

$$
\widehat{Z}(s)=\sum_{a \in A} t_{a}\left(A+B s+C s^{2}, s\right) f_{a}(s) .
$$

Then, by the Karush-Kuhn-Tucker necessary conditions for optimality of vectors $s^{*} \geq 0$, we know that the following must be true:

(i)

$$
\frac{\partial \widehat{Z}\left(s^{*}\right)}{\partial s^{i}}=0 \quad \text { if } s^{* i}>0, i=1, \ldots, n,
$$

(ii)

$$
\frac{\partial \widehat{Z}\left(s^{*}\right)}{\partial s^{i}} \geq 0 \quad \text { if } s^{* i}=0, \quad i=1, \ldots, n .
$$

So, taking the derivative with respect to $s$, we get

$$
\left.\frac{\partial}{\partial s^{i}}\left[\sum_{a} t_{a}\left(A+B s+C s^{2}, s\right)\left(A+B s+C s^{2}\right)\right]\right|_{s=s^{*}}
$$

$$
\begin{aligned}
=\left.\sum_{a} t_{a}\left(A+B s+C s^{2}, s\right) \cdot(B+2 C s)\right|_{s=s^{*}} \\
+\sum_{a}\left[\frac{\partial t_{a}\left(A+B s+C s^{2}, s\right)}{\partial\left(A+B s+C s^{2}\right)} \cdot(B+2 C s)\right. \\
\left.+\frac{\partial t_{a}\left(A+B s+C s^{2}, s\right)}{\partial s}\right]\left.\cdot\left(A+B s+C s^{2}\right)\right|_{s=s^{*}} .
\end{aligned}
$$

Further, we know

$$
\begin{gathered}
B=\left.D_{s} f\right|_{s=s^{*},} \\
C=\left.\frac{1}{2} \cdot H_{s} f\right|_{s=s^{*}}, \\
f(s)=A+B s+C s^{2} .
\end{gathered}
$$

So, substituting (29), we know

$$
\begin{gathered}
\left.\frac{\partial}{\partial s^{i}}\left[\sum_{a} t_{a}\left(A+B s+C s^{2}, s\right)\left(A+B s+C s^{2}\right)\right]\right|_{s=s^{*}} \\
=\left.\sum_{a} t_{a}(f(s), s) \cdot\left(D_{s} f+H_{s} f \cdot s\right)\right|_{s=s^{*}} \\
+\sum_{a}\left[\frac{\partial t_{a}(f(s), s)}{\partial f} \cdot\left(D_{s} f+H_{s} f \cdot s\right)\right. \\
\left.\quad+\frac{\partial t_{a}(f(s), s)}{\partial s}\right]\left.\cdot f_{a}\right|_{s=s^{*}, f=f^{*}} \\
=\left.\sum_{a} t_{a}(f(s), s) \cdot D_{s} f(s)\right|_{s=s^{*}} \\
+\sum_{a}\left[\frac{\partial t_{a}(f(s), s)}{\partial f} \cdot D_{s} f(s)\right. \\
\left.\quad+\frac{\partial t_{a}(f(s), s)}{\partial s}\right]\left.\cdot f_{a}\right|_{s=s^{*}, f=f^{*}} \\
=\left.\frac{\partial}{\partial s}\left[\sum_{a} t_{a}(f(s), s) \cdot f_{a}(s)\right]\right|_{s=s^{*}, f=f^{*}}
\end{gathered}
$$

So, we know if conditions (i) and (ii) are satisfied, then the following should also be satisfied:

(iii)

$$
\frac{\partial Z\left(s^{*}\right)}{\partial s^{i}}=0 \quad \text { if } s^{* i}>0, i=1, \ldots, n
$$

(iv)

$$
\frac{\partial Z\left(s^{*}\right)}{\partial s^{i}} \geq 0 \quad \text { if } s^{* i}=0, i=1, \ldots, n .
$$




\section{Numerical Example}

This section provides two numerical examples which illustrate the computation of second-order sensitivity and the performance of NLAA. The first example demonstrates the computation of second-order sensitivity in detail. The second example focuses on the speed of convergence between LAA and NLAA.

Example 1. The first example is chosen from Dickson [25] and Fisk [14]. The network topology is shown in Figure 1. The set of OD pairs is $\{(1,2),(3,4)\}$ and a signal exists at the intersection of arcs 1 and 3 . The cost functions used are

$$
t_{1}=\frac{f_{1}}{s_{1}}, \quad t_{2}=2 f_{2}, \quad t_{3}=\frac{2 f_{3}}{s_{3}},
$$

where $s_{a}$ denotes the green time on arc $a$ and the cycle time, $s_{1}+s_{3}$, is equal to 20 .

Additionally, the travel demand $T_{1}$ from node 1 to node 2 is 10 and the travel demand $T_{2}$ from node 3 to node 4 is 10. Table 1 lists the arc cost functions $t_{a}\left(f_{a}, s_{a}\right)$ and the system objective function $Z(s)$.

5.1. First-Order and Second-Order Sensitivity Formulas. In this example, $s_{3}$ can be replaced by $20-s_{1}$, and $s_{1}$ that is the only perturbation parameter (control variable) should be considered. Therefore, $\rho$ is equal to 1 . Together with (6) and (15), the first-order sensitivity with respect to $s_{1}$ can be rewritten as

$$
\begin{aligned}
& {\left[\begin{array}{l}
D_{s} f \\
D_{s} \mu
\end{array}\right]=U \cdot V} \\
& =\left[\begin{array}{ccccc}
\frac{s_{1}}{1+2 s_{1}} & \frac{-s_{1}}{1+2 s_{1}} & 0 & \frac{-2 s_{1}}{1+2 s_{1}} & 0 \\
\frac{-s_{1}}{1+2 s_{1}} & \frac{s_{1}}{1+2 s_{1}} & 0 & \frac{-s_{1}}{1+2 s_{1}} & 0 \\
0 & 0 & 0 & 0 & -1 \\
\frac{2 s_{1}}{1+2 s_{1}} & \frac{1}{1+2 s_{1}} & 0 & \frac{2}{1+2 s_{1}} & 0 \\
0 & 0 & 1 & 0 & \frac{-1}{-20+s_{1}}
\end{array}\right] \\
& \times\left[\begin{array}{c}
\frac{f_{1}}{s_{1}^{2}} \\
0 \\
\frac{-2 f_{3}}{\left(20-s_{1}\right)^{2}} \\
0 \\
0
\end{array}\right]=\left[\begin{array}{c}
\frac{f_{1}}{s_{1}\left(1+2 s_{1}\right)} \\
\frac{-f_{1}}{s_{1}\left(1+2 s_{1}\right)} \\
0 \\
\frac{2 f_{1}}{s_{1}\left(1+2 s_{1}\right)} \\
\frac{-2 f_{3}}{\left(20-s_{1}\right)^{2}}
\end{array}\right],
\end{aligned}
$$

TABLE 1: Arc cost functions and the system objective function in Example 1.

\begin{tabular}{lcc}
\hline \multicolumn{3}{c}{$t_{a}\left(f_{a}, s_{a}\right)=P_{a}+Q_{a}\left(f_{a} / s_{a}\right)$} \\
\hline$Z(s)=\sum_{a}\left(t_{a}\left(f_{a}, s_{a}\right) \cdot f_{a}\right)$ & \\
\hline Arc number & $P_{a}$ & $Q_{a}$ \\
\hline 1 & 2 & 1 \\
2 & 0 & 2 \\
3 & 0 & 2 \\
\hline
\end{tabular}

where

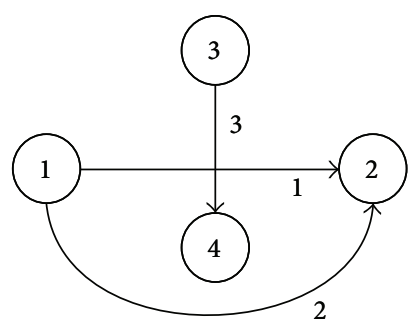

Figure 1: The network topology in Example 1.

$$
D_{s} f=\left[\begin{array}{c}
\frac{f_{1}}{s_{1}\left(1+2 s_{1}\right)} \\
\frac{-f_{1}}{s_{1}\left(1+2 s_{1}\right)} \\
0
\end{array}\right], \quad D_{s} \mu=\left[\begin{array}{c}
\frac{2 f_{1}}{s_{1}\left(1+2 s_{1}\right)} \\
\frac{-2 f_{3}}{\left(20-s_{1}\right)^{2}}
\end{array}\right] \text {. }
$$

In (36), the sensitivity information of arc flow represents the change of arc flow on $\operatorname{arc} a$, respectively, when the control variable $s_{1}$ increases one unit. Since $f_{1} \geq 0$ and $s_{1}>0$, the equilibrium flow on arc 1 will increase when $s_{1}$ increases one unit. In the meanwhile, the equilibrium flow on arc 2 will decrease. Because OD pair $(3,4)$ has only one path $(\operatorname{arc} 3)$, $s_{1}$ will not affect the equilibrium flow on arc 3 .

From Lemma 7, the second-order sensitivity with respect to control variable is

$$
\left[\begin{array}{l}
H_{s} f \\
H_{s} \mu
\end{array}\right]=\left(V^{T} \otimes I_{\left(\alpha+\alpha_{2}\right)}\right) D_{s} U+\left(I_{k} \otimes U\right) D_{s} V .
$$

By Theorem 4, $D_{s} U$ can be derived by the chain rule for matrix functions as follows:

$$
\begin{aligned}
D_{s} U & =\left(D_{f, \mu} U\right)\left[\begin{array}{l}
D_{s} f \\
D_{s} \mu
\end{array}\right]+\left(D_{s} U\right) \\
& =\left[\begin{array}{ll}
\frac{\partial \operatorname{vec} U}{\partial \operatorname{vec} f} & \frac{\partial \operatorname{vec} U}{\partial \operatorname{vec} \mu}
\end{array}\right]\left[\begin{array}{c}
D_{s} f \\
D_{s} \mu
\end{array}\right]+\left[\begin{array}{l}
\frac{\partial \operatorname{vec} U}{\partial \operatorname{vec} s_{1}}
\end{array}\right] .
\end{aligned}
$$


In this example, the matrix $U$ is only dependent on $s_{1}$. Hence, $D_{f, \mu} U=0$ and (38) can be rewritten as

$$
D_{s} U=\left[\frac{\partial \operatorname{vec} U}{\partial \operatorname{vec} s_{1}}\right]=\left[\begin{array}{l}
\frac{\partial U_{1}}{\partial s_{1}} \\
\frac{\partial U_{2}}{\partial s_{1}} \\
\frac{\partial U_{3}}{\partial s_{1}} \\
\frac{\partial U_{4}}{\partial s_{1}} \\
\frac{\partial U_{5}}{\partial s_{1}}
\end{array}\right],
$$

where $U_{j}$ represents the $j$ th column of matrix $U$, and

$$
\begin{aligned}
& \frac{\partial U_{1}}{\partial s_{1}}=\left[\begin{array}{c}
\frac{1}{\left(1+2 s_{1}\right)^{2}} \\
\frac{-1}{\left(1+2 s_{1}\right)^{2}} \\
0 \\
\frac{2}{\left(1+2 s_{1}\right)^{2}} \\
0
\end{array}\right], \quad \frac{\partial U_{2}}{\partial s_{1}}=\left[\begin{array}{c}
\frac{-1}{\left(1+2 s_{1}\right)^{2}} \\
\frac{1}{\left(1+2 s_{1}\right)^{2}} \\
0 \\
\frac{-2}{\left(1+2 s_{1}\right)^{2}} \\
0
\end{array}\right], \\
& \frac{\partial U_{3}}{\partial s_{1}}=\left[\begin{array}{l}
0 \\
0 \\
0 \\
0 \\
0
\end{array}\right], \quad \frac{\partial U_{4}}{\partial s_{1}}=\left[\begin{array}{c}
\frac{-2}{\left(1+2 s_{1}\right)^{2}} \\
\frac{2}{\left(1+2 s_{1}\right)^{2}} \\
0 \\
\frac{-4}{\left(1+2 s_{1}\right)^{2}} \\
0
\end{array}\right], \\
& \frac{\partial U_{5}}{\partial s_{1}}=\left[\begin{array}{c}
0 \\
0 \\
0 \\
0 \\
\frac{2}{\left(-20+s_{1}\right)^{2}}
\end{array}\right] \text {. }
\end{aligned}
$$

Similarly, $D_{s} V$ can be derived as

$$
\begin{aligned}
D_{s} V & =\left(D_{f, \mu} V\right)\left[\begin{array}{l}
D_{s} f \\
D_{s} \mu
\end{array}\right]+\left(D_{s} V\right) \\
& =\left[\begin{array}{ll}
\frac{\partial \operatorname{vec} V}{\partial \operatorname{vec} f} & \frac{\partial \operatorname{vec} V}{\partial \operatorname{vec} \mu}
\end{array}\right]\left[\begin{array}{l}
D_{s} f \\
D_{s} \mu
\end{array}\right]+\left[\begin{array}{ll}
\frac{\partial \operatorname{vec} V}{\partial \operatorname{vec} s_{1}}
\end{array}\right]
\end{aligned}
$$

$$
\begin{gathered}
=\left[\begin{array}{ccccc}
\frac{1}{s_{1}^{2}} & 0 & 0 & 0 & 0 \\
0 & 0 & 0 & 0 & 0 \\
0 & 0 & \frac{-2}{\left(20-s_{1}\right)^{2}} & 0 & 0 \\
0 & 0 & 0 & 0 & 0 \\
0 & 0 & 0 & 0 & 0
\end{array}\right]\left[\begin{array}{c}
\frac{f_{1}}{s_{1}\left(1+2 s_{1}\right)} \\
\frac{-f_{1}}{s_{1}\left(1+2 s_{1}\right)} \\
0 \\
\frac{2 f_{1}}{s_{1}\left(1+2 s_{1}\right)} \\
\frac{-2 f_{3}}{\left(20-s_{1}\right)^{2}}
\end{array}\right] \\
+\left[\begin{array}{c}
\frac{-2 f_{1}}{s_{1}^{3}} \\
0 \\
\frac{-4 f_{3}}{\left(20-s_{1}\right)^{3}} \\
0 \\
0
\end{array}\right]=\left[\begin{array}{c}
\frac{f_{1}}{s_{1}^{3}\left(1+2 s_{1}\right)}-\frac{2 f_{1}}{s_{1}^{3}} \\
0 \\
\frac{-4 f_{3}}{\left(20-s_{1}\right)^{3}} \\
0 \\
0
\end{array}\right] .
\end{gathered}
$$

According to Definition 1, (39), (40), and (38), (37) can be rewritten as

$$
\begin{aligned}
& {\left[\begin{array}{c}
H_{s} f \\
H_{s} \mu
\end{array}\right]=\left(V^{T} \otimes I_{5}\right) D_{s} U+\left(I_{1} \otimes U\right) D_{s} V} \\
& =\left[\begin{array}{c}
\frac{f_{1}}{s_{1}^{2}} \cdot I_{5} \\
0 \cdot I_{5} \\
\frac{-2 f_{3}}{\left(20-s_{1}\right)^{2}} \cdot I_{5} \\
0 \cdot I_{5} \\
0 \cdot I_{5}
\end{array}\right]^{T}\left[\begin{array}{l}
\frac{\partial U_{1}}{\partial s_{1}} \\
\frac{\partial U_{2}}{\partial s_{1}} \\
\frac{\partial U_{3}}{\partial s_{1}} \\
\frac{\partial U_{4}}{\partial s_{1}} \\
\frac{\partial U_{5}}{\partial s_{1}}
\end{array}\right] \\
& +(1 \cdot U)\left[\begin{array}{c}
\frac{f_{1}}{s_{1}^{3}\left(1+2 s_{1}\right)}-\frac{2 f_{1}}{s_{1}^{3}} \\
0 \\
\frac{-4 f_{3}}{\left(20-s_{1}\right)^{3}} \\
0 \\
0
\end{array}\right] \\
& =\left[\begin{array}{c}
\frac{-4 f_{1}}{s_{1}\left(1+2 s_{1}\right)^{2}} \\
\frac{4 f_{1}}{s_{1}\left(1+2 s_{1}\right)^{2}} \\
0 \\
\frac{-8 f_{1}}{s_{1}\left(1+2 s_{1}\right)^{2}} \\
\frac{4 f_{3}}{\left(-20+s_{1}\right)^{3}}
\end{array}\right] .
\end{aligned}
$$


Therefore,

$$
H_{s} f=\left[\begin{array}{c}
\frac{-4 f_{1}}{s_{1}\left(1+2 s_{1}\right)^{2}} \\
\frac{4 f_{1}}{s_{1}\left(1+2 s_{1}\right)^{2}} \\
0
\end{array}\right], \quad H_{s} \mu=\left[\begin{array}{c}
\frac{-8 f_{1}}{s_{1}\left(1+2 s_{1}\right)^{2}} \\
\frac{4 f_{3}}{\left(-20+s_{1}\right)^{3}}
\end{array}\right]
$$

5.2. Computational Results of LAA and NLAA. Based on (36) and (43), the first differential and the second differential of equilibrium arc flow $f_{a}$ can be obtained by (14). At iteration $k$,

$$
\begin{aligned}
& B s^{k+1}=d f\left(s_{0} ; s\right)=\left.D_{s} f\right|_{f=f^{k}, s_{1}=s_{1}^{k}} \cdot\left(s_{1}^{k+1}-s_{1}^{k}\right) \\
& =\left[\begin{array}{c}
\frac{f_{1}^{k}}{s_{1}^{k}\left(1+2 s_{1}^{k}\right)} \\
\frac{-f_{1}^{k}}{s_{1}^{k}\left(1+2 s_{1}^{k}\right)} \\
0
\end{array}\right] \cdot\left(s_{1}^{k+1}-s_{1}^{k}\right) \\
& =\left[\begin{array}{c}
\frac{f_{1}^{k}\left(s_{1}^{k+1}-s_{1}^{k}\right)}{s_{1}^{k}\left(1+2 s_{1}^{k}\right)} \\
\frac{-f_{1}^{k}\left(s_{1}^{k+1}-s_{1}^{k}\right)}{s_{1}^{k}\left(1+2 s_{1}^{k}\right)} \\
0
\end{array}\right] \\
& C\left(s^{k+1}\right)^{2}=\frac{1}{2} d^{2} f\left(s_{0} ; s\right) \\
& =\left.\frac{1}{2}\left(\left(s_{1}^{k+1}-s_{1}^{k}\right)^{T} \otimes I_{3}\right) \cdot H_{s} f\right|_{f=f^{k}, s_{1}=s_{1}^{k}} \\
& \cdot\left(s_{1}^{k+1}-s_{1}^{k}\right) \\
& =\frac{1}{2}\left(\left(s_{1}^{k+1}-s_{1}^{k}\right) \otimes\left[\begin{array}{lll}
1 & 0 & 0 \\
0 & 1 & 0 \\
0 & 0 & 1
\end{array}\right]\right) \cdot\left[\begin{array}{c}
\frac{-4 f_{1}^{k}}{s_{1}^{k}\left(1+2 s_{1}^{k}\right)^{2}} \\
\frac{4 f_{1}^{k}}{s_{1}^{k}\left(1+2 s_{1}^{k}\right)^{2}} \\
0
\end{array}\right] \\
& \cdot\left(s_{1}^{k+1}-s_{1}^{k}\right) \\
& =\left[\begin{array}{c}
\frac{-4 f_{1}^{k}\left(s_{1}^{k+1}-s_{1}^{k}\right)^{2}}{2 s_{1}^{k}\left(1+2 s_{1}^{k}\right)^{2}} \\
\frac{4 f_{1}^{k}\left(s_{1}^{k+1}-s_{1}^{k}\right)^{2}}{2 s_{1}^{k}\left(1+2 s_{1}^{k}\right)^{2}} \\
0
\end{array}\right] .
\end{aligned}
$$

In this example, both LAA and NLAA are implemented in the MATLAB environment. Set $\delta=0.001$ and the initial $s_{1}=10$; Table 2 lists the computational results of LAA and NLAA approaches, and it shows that NLAA is more efficient than LAA.
TABLE 2: Computational results of LAA and NLAA in Example 1.

\begin{tabular}{lcccccc}
\hline \multirow{2}{*}{ Iteration } & \multicolumn{3}{c}{ LAA } & & \multicolumn{3}{c}{ NLAA } \\
& $f_{1}$ & $s_{1}$ & $Z$ & $f_{1}$ & $s_{1}$ & $Z$ \\
\hline 1 & 8.44693 & 7.63647 & 47.23792 & 8.45125 & 7.70052 & 47.23576 \\
2 & 8.45366 & 7.73667 & 47.23553 & 8.45326 & 7.73055 & 47.23552 \\
3 & 8.45323 & 7.73019 & 47.23552 & 8.45326 & 7.73056 & 47.23552 \\
4 & 8.45325 & 7.73040 & 47.23552 & & & \\
\hline
\end{tabular}

Example 2. This example is a simplified real network which represents the afternoon rush hour traffic between the working area Hsinchu Science-Based Industrial Park (HSIP) and the residential area Jhubei city. The network topology follows Figure 2. In this period, there is a large amount of travel demand from HSIP (node 1) to Jhubei city (node 16). There are two parallel paths from HSIP to Jhubei city. One is a freeway (arc 2-arc 4-arc 16) and the other is a highway with 5 signal-controlled intersections (arc 1-arc 6-arc 8-arc 10 -arc 12-arc 14). The objective of this problem is to find the optimal signal settings which minimize the system cost. The arc cost functions $t_{a}\left(f_{a}, s_{a}\right)$ and the system objective function $Z(s)$ are listed in Table 3. Table 4 lists the origindestination demand. For the signal-controlled intersections, the arcs entering the same intersection share the same cycle time and the minimum green time for each approach is $10 \mathrm{sec}$. In this example, we set $\delta=0.1$ and the initial $s_{a}=$ $C y c_{a} / 2$ for each signalized arc. Table 5 lists the computational results of LAA and NLAA, respectively. Two parallel paths from node 1 to node 16 (2-4-16 and 1-6-8-10-12-14) have the same equilibrium travel time $13.4069 \mathrm{~min}$. Table 5 shows that NLAA only takes $6 \%$ iterations, compared with LAA, to attain the same level of precision. Figure 3 shows the convergence curves of LAA and NLAA, respectively. The convergence rate of LAA is slower than NLAA due to the zigzag effect. Compared with Example 1, NLAA has more improvement in the speed of convergence than in Example 2. It may imply that NLAA is more efficient to deal with more nonlinear problems.

\section{Conclusions}

The key information to solve the equilibrium network signal control problem (ENSCP) is the reaction function of the lower-level problem. Because the reaction function cannot be obtained explicitly, the sensitivity information of equilibrium network flows is used to approximate it. Based on the first-order sensitivity formula and the matrix calculus, this paper first presents the general form of the second-order sensitivity formula for equilibrium network flows. With the second-order sensitivity formula, the reaction function can be approximated more accurately by a nonlinear function. From HSIP to Jhubei city, a simplified real network example demonstrates the speed of convergence between LAA and NLAA. The NLAA has significant improvement in solving the ENSCP with complicated arc cost functions; in this example, the NLAA only takes $6 \%$ iterations to attain the same level of precision. 


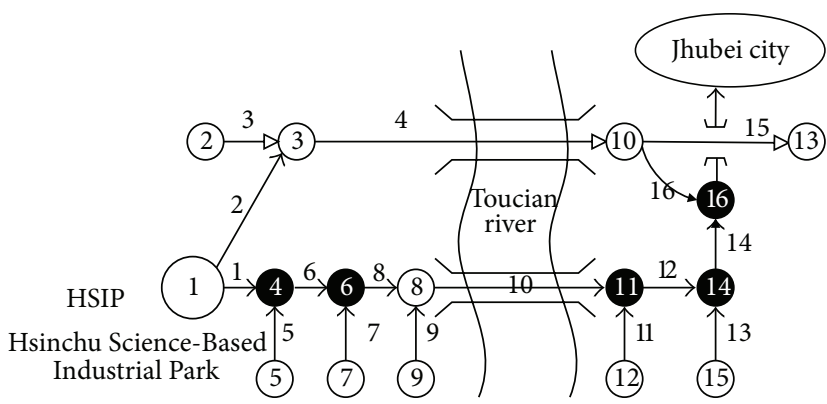

- Signal-controlled intersection

$\rightarrow$ Free way

FIgURE 2: The network topology in Example 2.

TABle 3: Arc cost functions and the system objective function in Example 2.

\begin{tabular}{|c|c|c|c|c|c|}
\hline \multicolumn{2}{|c|}{ Signalized arc cost function } & & ${ }_{a}\left(f_{a}, s_{a}\right)$ & $\alpha_{a}\left(\frac{f_{a}}{C_{a}\left(s_{a} / \mathrm{Cyc}\right.}\right.$ & \\
\hline \multicolumn{2}{|c|}{ Nonsignalized arc cost function } & \multicolumn{4}{|c|}{$t_{a}\left(f_{a}, s_{a}\right)=t_{0 a}\left(1+\alpha_{a}\left(\frac{f_{a}}{C_{a}}\right)^{\beta_{a}}\right)$} \\
\hline \multicolumn{2}{|c|}{ System objective function } & \multicolumn{4}{|c|}{$Z(s)=\sum_{a}\left(t_{a}\left(f_{a}, s_{a}\right) \cdot f_{a}\right)$} \\
\hline Arc number & $t_{0 a}(\min )$ & $\alpha_{a}$ & $\beta_{a}$ & $C_{a}(\mathrm{veh} / \mathrm{min})$ & $\mathrm{Cyc}_{a}(\mathrm{sec})$ \\
\hline 1 & 1.8545 & 0.9200 & 3.5800 & 56.6667 & 300 \\
\hline 2 & 0.8667 & 0.9200 & 3.5800 & 40.0000 & - \\
\hline 3 & 1.8000 & 1.2700 & 3.9600 & 115.0000 & - \\
\hline 4 & 2.2364 & 1.2700 & 3.9600 & 115.0000 & - \\
\hline 5 & 0.2945 & 1.2100 & 2.3900 & 28.3333 & 300 \\
\hline 6 & 0.1964 & 1.4200 & 2.3200 & 85.0000 & 300 \\
\hline 7 & 0.3818 & 0.8600 & 4.3400 & 85.0000 & 300 \\
\hline 8 & 1.0154 & 1.2700 & 3.9600 & 68.3333 & - \\
\hline 9 & 1.0000 & 1.2100 & 2.3900 & 20.0000 & - \\
\hline 10 & 1.0154 & 1.2700 & 3.9600 & 68.3333 & 180 \\
\hline 11 & 0.3273 & 0.9200 & 3.5800 & 56.6667 & 180 \\
\hline 12 & 0.9818 & 1.4200 & 2.3200 & 85.0000 & 150 \\
\hline 13 & 0.6545 & 0.8600 & 4.3400 & 113.3333 & 150 \\
\hline 14 & 1.2000 & 1.5000 & 2.4400 & 113.3333 & 150 \\
\hline 15 & 3.8727 & 1.2700 & 3.9600 & 115.0000 & - \\
\hline 16 & 0.4909 & 0.9200 & 3.5800 & 40.0000 & 150 \\
\hline
\end{tabular}

TABle 4: Origin-destination demand table in Example 2 (unit: veh/hr).

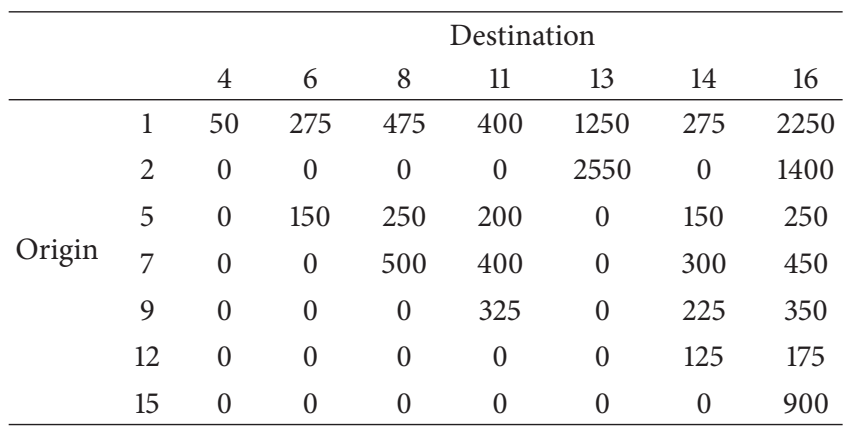

This study focuses on the NLAA and a simplified delay formula is adopted to reflect the influence of traffic congestion. Practically, a traffic propagation model, such as TRANSYT model, should be included when solving the ENSCP. Since the derivatives of TRANSYT model have been obtained explicitly [4, 42], it can be extended to second-order derivatives and applied to NLAA in the future research.

Compared with LAA, the number of multiplications for matrix multiplication is greatly increasing in NLAA due to the Kronecker-product operation. NLAA has polynomial complexity with the network size and the number of perturbation parameters because of the property of the Kronecker product. There is still opportunity to improve the 
TABLE 5: Computational results of LAA and NLAA in Example 2.

\begin{tabular}{|c|c|c|c|c|c|c|}
\hline \multirow{2}{*}{ Arc number } & \multicolumn{3}{|c|}{ LAA } & \multicolumn{3}{|c|}{ NLAA } \\
\hline & $s_{a}(\mathrm{sec})$ & $f_{a}(\mathrm{veh} / \mathrm{min})$ & $t_{a}(\min )$ & $s_{a}(\mathrm{sec})$ & $f_{a}(\mathrm{veh} / \mathrm{min})$ & $t_{a}(\min )$ \\
\hline 1 & 195.9103 & 31.4891 & 2.8118 & 195.8881 & 31.4901 & 2.8123 \\
\hline 2 & - & 51.4275 & 2.8272 & - & 51.4266 & 2.8271 \\
\hline 3 & - & 65.8333 & 2.0510 & - & 65.8333 & 2.0510 \\
\hline 4 & - & 117.2609 & 5.3043 & - & 117.2599 & 5.3042 \\
\hline 5 & 104.0897 & 16.6667 & 1.5529 & 104.1119 & 16.6667 & 1.5522 \\
\hline 6 & 181.5056 & 47.3225 & 0.4263 & 181.4711 & 47.3234 & 0.4265 \\
\hline 7 & 118.4944 & 27.5000 & 0.5199 & 118.5289 & 27.5000 & 0.5197 \\
\hline 8 & - & 67.7391 & 2.2611 & - & 67.7401 & 2.2612 \\
\hline 9 & - & 15.0000 & 1.6084 & - & 15.0000 & 1.6084 \\
\hline 10 & 167.0451 & 62.3225 & 2.2192 & 167.0853 & 62.3234 & 2.2181 \\
\hline 11 & 12.9549 & 5.0000 & 0.9517 & 12.9147 & 5.0000 & 0.9587 \\
\hline 12 & 123.8799 & 45.2391 & 1.4849 & 123.8808 & 45.2401 & 1.4849 \\
\hline 13 & 26.1201 & 15.0000 & 0.8256 & 26.1192 & 15.0000 & 0.8256 \\
\hline 14 & 45.4083 & 42.3225 & 4.2042 & 45.4092 & 42.3234 & 4.2042 \\
\hline 15 & - & 63.3333 & 4.3361 & - & 63.3333 & 4.3361 \\
\hline 16 & 104.5917 & 53.9275 & 5.2760 & 104.5908 & 53.9266 & 5.2759 \\
\hline Iteration number & & 101 & & & 6 & \\
\hline Objective value $(Z)$ & & 2188.2886 & & & 2188.2404 & \\
\hline
\end{tabular}

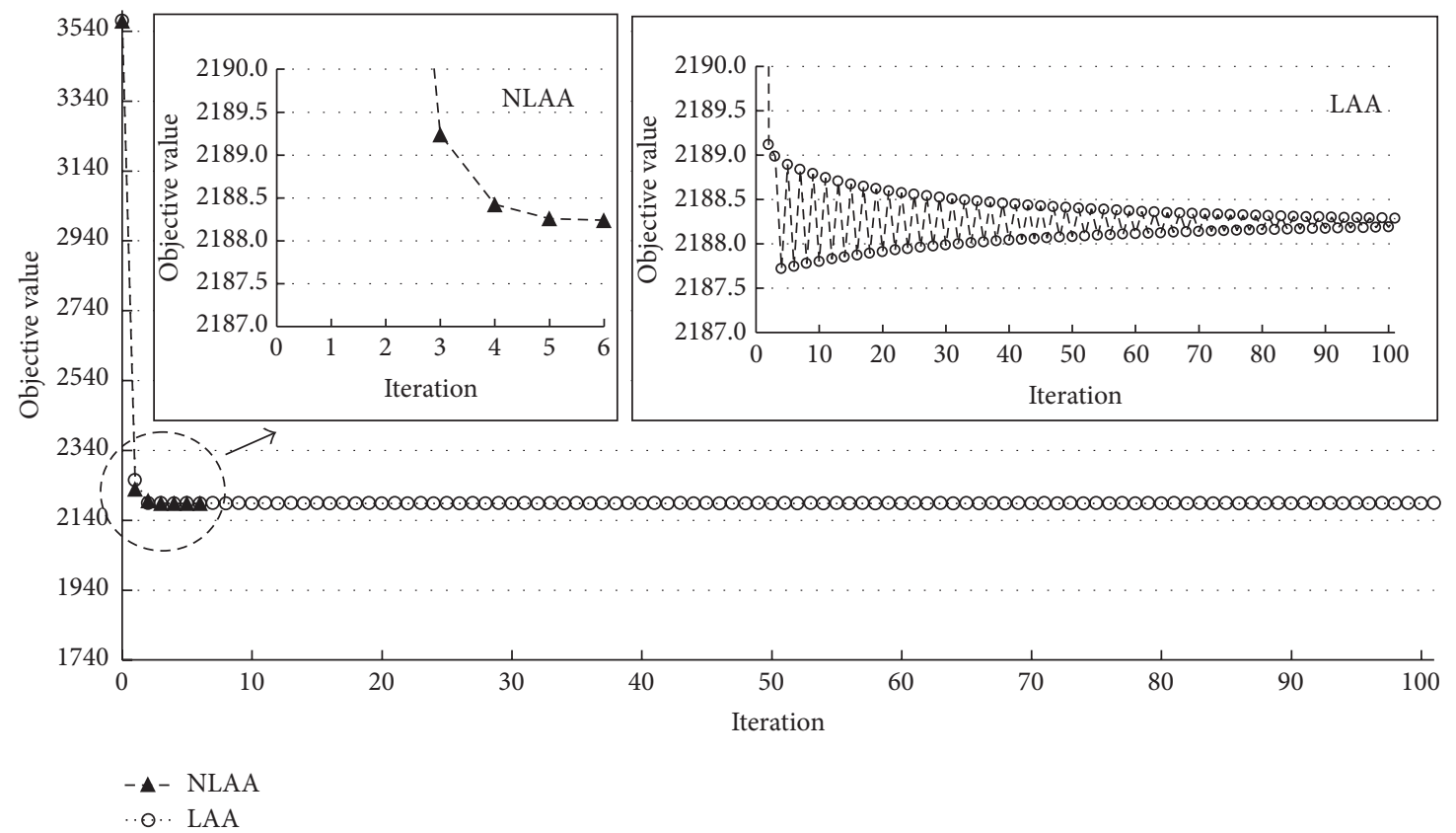

FIgURE 3: The convergence curves of LAA and NLAA in Example 2.

computing efficiency through adopting effective Kroneckerproduct algorithms.

\section{Conflict of Interests}

The authors declare that there is no conflict of interests regarding the publication of this paper.

\section{Acknowledgments}

This research was partially supported by the National Science Council, Taiwan; Ministry of Transportation and Communications, Taiwan; Chunghwa Telecom Laboratories, Taiwan, under contract number NSC-100-2221-E-009-119, MOTC-STAO-101-02, MOTC-STAO-102-02, TL-103-G109, 
respectively. The authors also acknowledge the reviewer's good suggestions for the further research topics.

\section{References}

[1] R. E. Allsop, "Evolving applications of mathematical optimization in design and operation of individual signal-controlled road junctions," in Mathematics in Transport Planning and Control, J. D. Griffiths, Ed., pp. 1-24, Clarendon Press, Oxford, UK, 1992.

[2] J. P. Silcock and A. Sang, "SIGSIGN: a phase-based optimisation program for individual signal-controlled junctions," Traffic Engineering and Control, vol. 31, no. 5, pp. 291-298, 1990.

[3] S. C. Wong, "Group-based optimisation of signal timings using the TRANSYT traffic model," Transportation Research B: Methodological, vol. 30, no. 3, pp. 217-244, 1996.

[4] S.-W. Chiou, "TRANSYT derivatives for area traffic control optimisation with network equilibrium flows," Transportation Research B: Methodological, vol. 37, no. 3, pp. 263-290, 2003.

[5] H. Ceylan and M. G. H. Bell, "Reserve capacity for a road network under optimized fixed time traffic signal control," Journal of Intelligent Transportation Systems: Technology, Planning, and Operations, vol. 8, no. 2, pp. 87-99, 2004.

[6] K. Tang and H. Nakamura, "Investigating operational benefits of group-based signal control in Japan through a stochastic approach," International Journal of Intelligent Transportation Systems Research, vol. 9, no. 2, pp. 71-81, 2011.

[7] S. C. Wong and C. Yang, "Iterative group-based signal optimization scheme for traffic equilibrium networks," Journal of Advanced Transportation, vol. 33, no. 2, pp. 201-217, 1999.

[8] T. L. Friesz, "Transportation network equilibrium, design and aggregation: key developments and research opportunities," Transportation Research A: General, vol. 19, no. 5-6, pp. 413-427, 1985.

[9] D. Braess, "Über ein paradoxon aus der verkehrsplanung," Mathematical Methods of Operations Research, vol. 12, no. 1, pp. 258-268, 1968.

[10] L. J. Leblanc, "An algorithm for the discrete network design problem," Transportation Science, vol. 9, no. 3, pp. 183-199, 1975.

[11] M. Abdulaal and L. J. LeBlanc, "Continuous equilibrium network design models," Transportation Research B: Methodological, vol. 13, no. 1, pp. 19-32, 1979.

[12] R. E. Allsop, "Some possibilities for using traffic control to influence trip distribution and route choice," in Proceedings of the 6th International Symposium on Transportation and Traffic Theory, pp. 345-373, Sydney, Australia, 1974.

[13] N. H. Gartner, S. B. Gershwin, J. D. C. Little, and P. Ross, "Pilot study of computer-based urban traffic management," Transportation Research B: Methodological, vol. 14, no. 1-2, pp. 203-217, 1980.

[14] C. S. Fisk, "Game theory and transportation systems modelling," Transportation Research B: Methodological, vol. 18, no. 4-5, pp. 301-313, 1984.

[15] W. Mao, F. Qin, Y. Hu, and Z. Tan, "Joint implementation of signal control and congestion pricing in transportation network," Journal of Applied Mathematics, vol. 2013, Article ID 315752, 6 pages, 2013.

[16] O. Baskan, "Determining optimal link capacity expansions in road networks using Cuckoo search algorithm with Lévy flights," Journal of Applied Mathematics, vol. 2013, Article ID 718015, 11 pages, 2013.
[17] P. Marcotte, "Network optimization with continuous control parameters," Transportation Science, vol. 17, no. 2, pp. 181-197, 1983.

[18] Y. Sheffi and W. B. Powell, "Optimal signal setting over transportation networks," Journal of Transportation Engineering, vol. 109, no. 6, pp. 824-839, 1983.

[19] B. G. Heydecker and T. K. Khoo, "The equilibrium network design problem," in Proceedings of the AIRO Conference on Models and Methods for Decision Support, pp. 587-602, Sorrento, Italy, 1990.

[20] M. J. Smith and T. van Vuren, "Traffic equilibrium with responsive traffic control," Transportation Science, vol. 27, no. 2, pp. 118-132, 1993.

[21] N. H. Tan, S. B. Gershwin, and M. Athans, "Hybrid optimization in urban transport networks," MIT Report Dot-TSC-RSPA-797, 1979.

[22] G. E. Cantarella, G. Improta, and A. Sforza, "Iterative procedure for equilibrium network traffic signal setting," Transportation Research A: General, vol. 25, no. 5, pp. 241-249, 1991.

[23] M. J. Smith, T. van Vuren, B. J. Heydecker, and D. van Vliet, "The interaction between signal control policies and route choices," in Proceedings of the 10th International Symposium on Transportation and Traffic Theory, pp. 319-338, Cambridge, Mass, USA, 1987.

[24] T. van Vuren and D. van Vliet, Route Choice and Signal Control, Athenaeum Press, Newcastle, UK, 1992.

[25] T. J. Dickson, "A note on traffic assignment and signal timings in a signal-controlled road network," Transportation Research B: Methodological, vol. 15, no. 4, pp. 267-271, 1981.

[26] T. L. Friesz, R. L. Tobin, H.-J. Cho, and N. J. Mehta, "Sensitivity analysis based heuristic algorithms for mathematical programs with variational inequality constraints," Mathematical Programming, vol. 48, no. 1-3, pp. 265-284, 1990.

[27] T.-J. Kim, Advanced Transport and Spatial Systems Models: Applications to Korea, Springer, New York, NY, USA, 1990.

[28] H. Yang and S. Yagar, "Traffic assignment and signal control in saturated road networks," Transportation Research A: General, vol. 29, no. 2, pp. 125-139, 1995.

[29] S. C. Wong and H. Yang, "Reserve capacity of a signal-controlled road network," Transportation Research B: Methodological, vol. 31, no. 5, pp. 397-402, 1997.

[30] H. Yang and M. G. H. Bell, "Models and algorithms for road network design: a review and some new developments," Transport Reviews, vol. 18, no. 3, pp. 257-278, 1998.

[31] H.-J. Cho, "Generalized inverse approach to sensitivity analysis of equilibrium network flow," Transportation Planning Journal, vol. 20, pp. 1-14, 1991 (Chinese).

[32] H.-J. Cho, T. E. Smith, and T. L. Friesz, "A reduction method for local sensitivity analyses of network equilibrium arc flows," Transportation Research B: Methodological, vol. 34, no. 1, pp. 3151, 2000.

[33] M. Patriksson, "Sensitivity analysis of traffic equilibria," Transportation Science, vol. 38, no. 3, pp. 258-281, 2004.

[34] M. Josefsson and M. Patriksson, "Sensitivity analysis of separable traffic equilibrium equilibria with application to bilevel optimization in network design," Transportation Research B: Methodological, vol. 41, no. 1, pp. 4-31, 2007.

[35] H. Yang and M. G. H. Bell, "Sensitivity analysis of network traffic equilibria revisited: the corrected approach," in Proceedings of the 4th IMA International Conference on Mathematics in Transport, pp. 373-396, London, UK, 2007. 
[36] H.-J. Cho and S.-C. Lo, "Solving bilevel network design problem using a linear reaction function without nondegeneracy assumption," Transportation Research Record, vol. 1667, pp. 96106, 1999.

[37] H.-J. Cho and G.-S. Lin, "Nonlinear approximation of reaction function for Stackelberg game in transportation network design," Mathematical and Computer Modelling. In press.

[38] S.-W. Chiou, "An iterative scheme for signal settings and network flows," Applied Mathematics and Computation, vol. 189, no. 2, pp. 1808-1815, 2007.

[39] S.-W. Chiou, "A hybrid approach for optimal design of signalized road network," Applied Mathematical Modelling, vol. 32, no. 2, pp. 195-207, 2008.

[40] S.-W. Chiou, "A non-smooth model for signalized road network design problems," Applied Mathematical Modelling, vol. 32, no. 7, pp. 1179-1190, 2008.

[41] S.-W. Chiou, "Optimization for signal setting problems using non-smooth techniques," Information Sciences, vol. 179, no. 17, pp. 2985-2996, 2009.

[42] S. C. Wong, "Derivatives of the performance index for the traffic model from TRANSYT," Transportation Research B: Methodological, vol. 29, no. 5, pp. 303-327, 1995.

[43] S.-W. Chiou, "Joint optimization for area traffic control and network flow," Computers \& Operations Research, vol. 32, no. 11, pp. 2821-2841, 2005.

[44] S.-W. Chiou, "An efficient algorithm for optimal design of area traffic control with network flows," Applied Mathematical Modelling, vol. 33, no. 6, pp. 2710-2722, 2009.

[45] J. Wardrop, "Some theoretical aspects of road traffic research," Proceedings of the Institute of Civil Engineers II, vol. 1, no. 3, pp. 325-378, 1952.

[46] S. C. Dafermos, "Traffic equilibrium and variational inequalities," Transportation Science, vol. 14, no. 1, pp. 42-54, 1980.

[47] R. L. Tobin and T. L. Friesz, "Sensitivity analysis for equilibrium network flow," Transportation Science, vol. 22, no. 4, pp. 242250, 1988.

[48] M. G. H. Bell and Y. Iida, Transportation Network Analysis, John Wiley \& Sons, Chichester, UK, 1997.

[49] J. R. Magnus and H. Neudecker, "Matrix differential calculus with applications to simple, Hadamard, and Kronecker products," Journal of Mathematical Psychology, vol. 29, no. 4, pp. 474492, 1985.

[50] J. R. Magnus and H. Neudecker, Matrix Differential Calculus with Applications in Statistics and Econometrics, John Wiley \& Sons, Chichester, UK, 1999.

[51] T. L. Friesz, H.-J. Cho, N. J. Mehta, R. L. Tobin, and G. Anandalingam, "Simulated annealing approach to the network design problem with variational inequality constraints," Transportation Science, vol. 26, no. 1, pp. 18-26, 1992. 


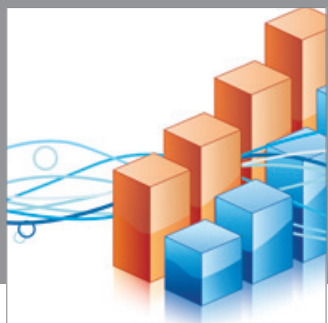

Advances in

Operations Research

mansans

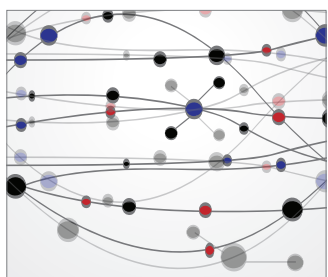

The Scientific World Journal
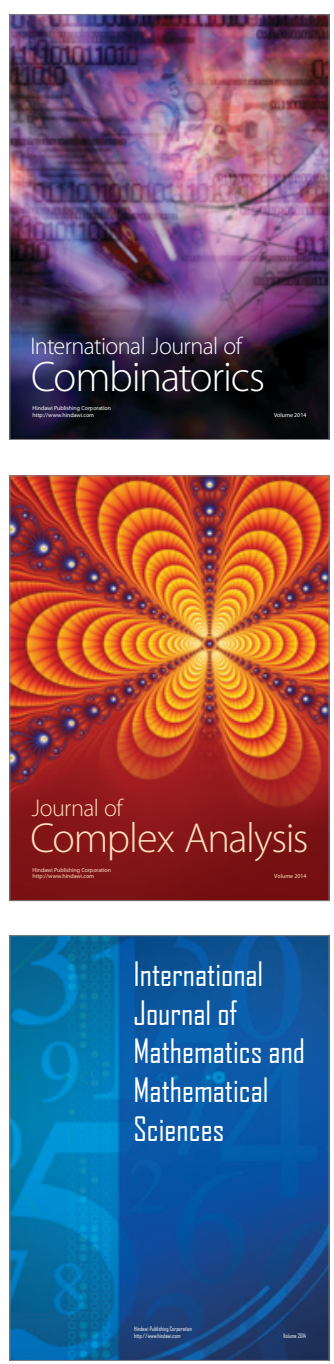
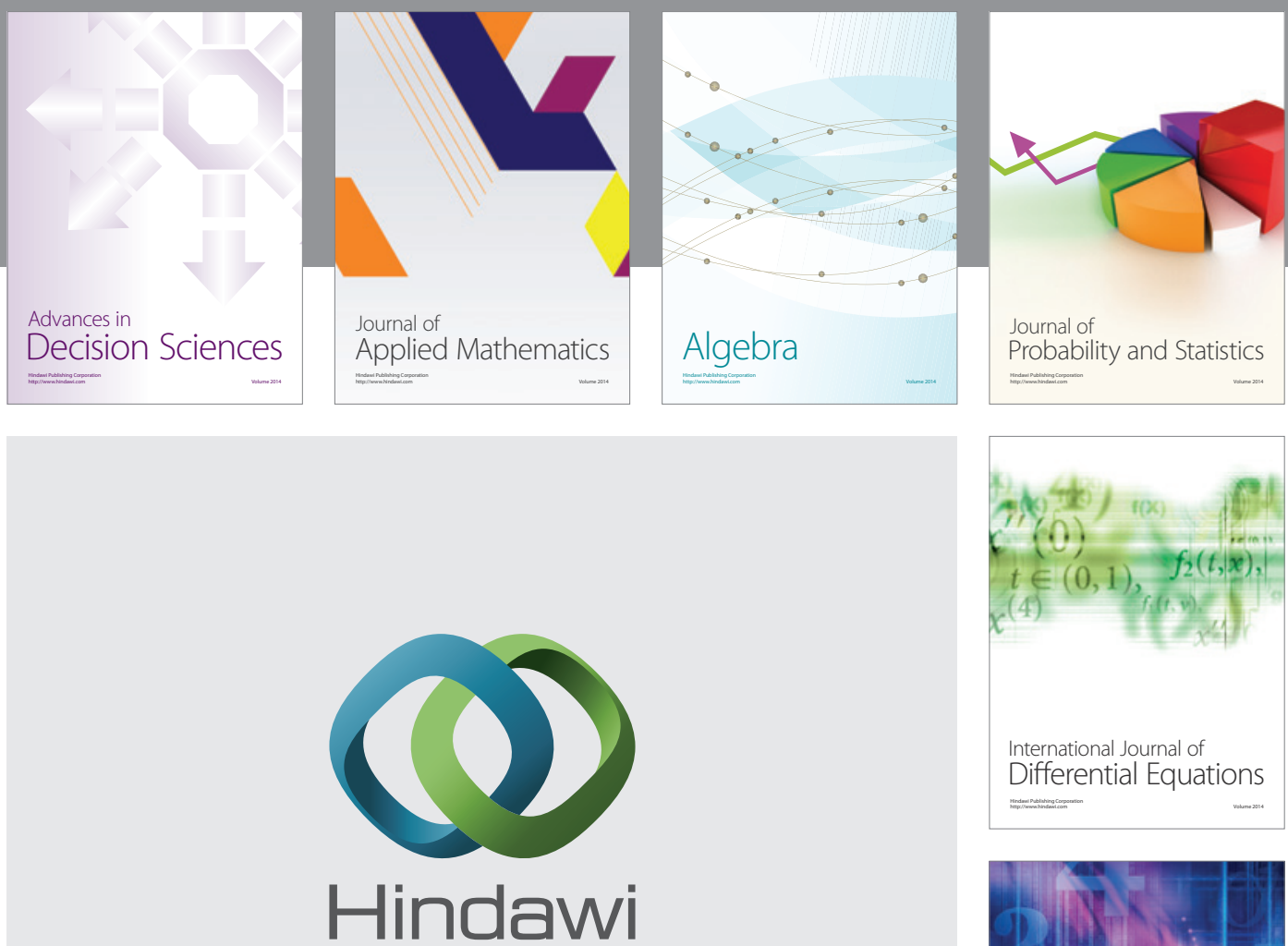

Submit your manuscripts at http://www.hindawi.com
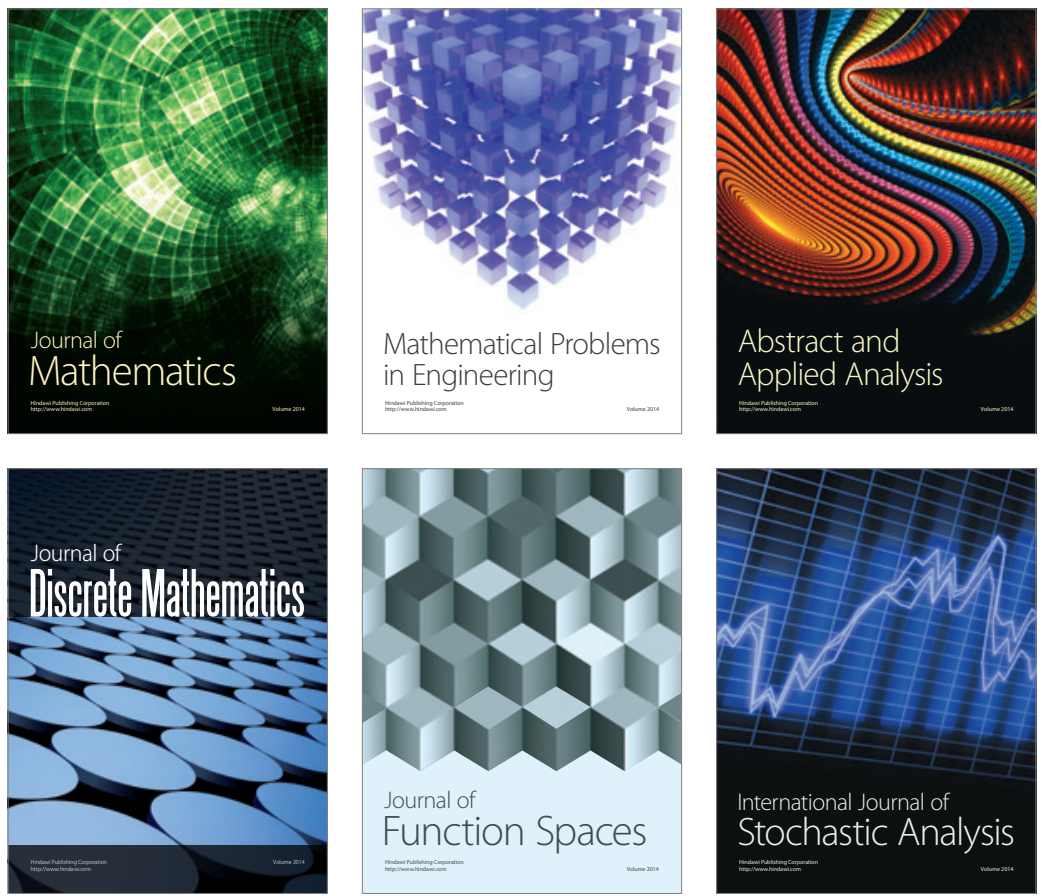

Journal of

Function Spaces

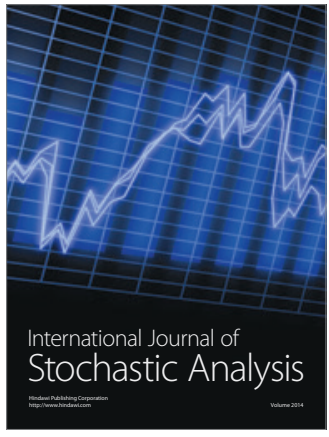

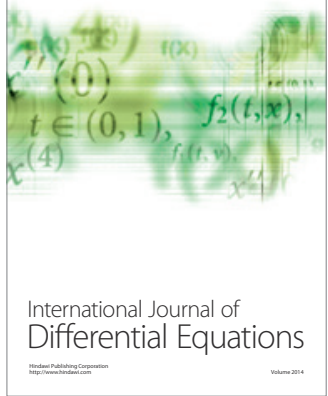
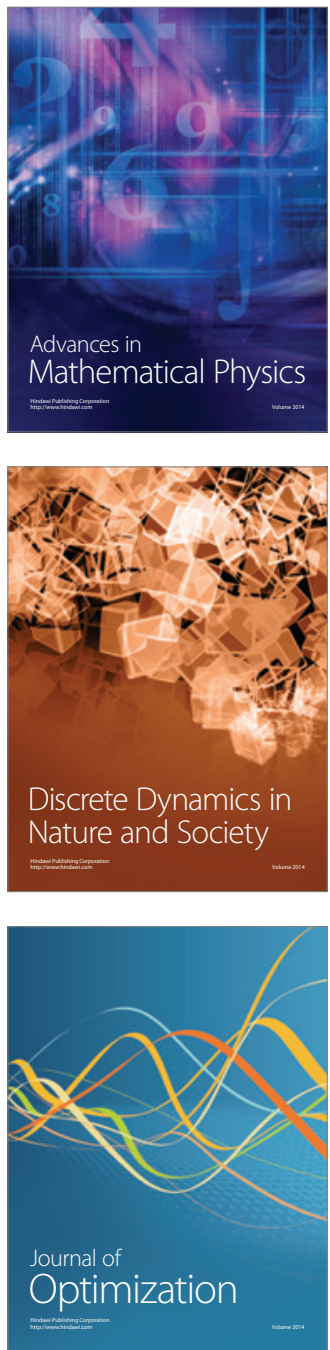